\title{
Sympatric chimpanzees and gorillas harbor convergent gut microbial communities
}

\author{
Andrew H. Moeller, ${ }^{1}$ Martine Peeters, ${ }^{2}$ Jean-Basco Ndjango, ${ }^{3}$ Yingying $\mathrm{Li}^{4}$ \\ Beatrice H. Hahn, ${ }^{4}$ and Howard Ochman ${ }^{1,5}$ \\ ${ }^{1}$ Department of Ecology and Evolutionary Biology, Yale University, New Haven, Connecticut 06511, USA; ${ }^{2}$ Institut de Recherche \\ pour le Développement (IRD) and University of Montpellier 1, 34394 Montpellier Cedex 5, France; ${ }^{3}$ Faculties of Sciences, University \\ of Kisangani, Kisangani, BP 2012, Democratic Republic of the Congo; ${ }^{4}$ Departments of Medicine and Microbiology, Perelman \\ School of Medicine, University of Pennsylvania, Philadelphia, Pennsylvania 19104, USA
}

\begin{abstract}
The gut microbial communities within great apes have been shown to reflect the phylogenetic history of their hosts, indicating codiversification between great apes and their gut microbiota over evolutionary timescales. But because the great apes examined to date represent geographically isolated populations whose diets derive from different sources, it is unclear whether this pattern of codiversification has resulted from a long history of coadaptation between microbes and hosts (heritable factors) or from the ecological and geographic separation among host species (environmental factors). To evaluate the relative influences of heritable and environmental factors on the evolution of the great ape gut microbiota, we assayed the gut communities of sympatric and allopatric populations of chimpanzees, bonobos, and gorillas residing throughout equatorial Africa. Comparisons of these populations revealed that the gut communities of different host species can always be distinguished from one another but that the gut communities of sympatric chimpanzees and gorillas have converged in terms of community composition, sharing on average $53 \%$ more bacterial phylotypes than the gut communities of allopatric hosts. Host environment, independent of host genetics and evolutionary history, shaped the distribution of bacterial phylotypes across the Bacteroidetes, Firmicutes, Proteobacteria, and Actinobacteria, the four most common phyla of gut bacteria. Moreover, the specific patterns of phylotype sharing among hosts suggest that chimpanzees living in sympatry with gorillas have acquired bacteria from gorillas. These results indicate that geographic isolation between host species has promoted the evolutionary differentiation of great ape gut bacterial communities.
\end{abstract}

[Supplemental material is available for this article.]

The compositions of the gut microbial communities harbored by great apes reflect the phylogeny of their hosts in a manner suggesting that host species and their gut microbiota have codiversified over evolutionary timescales (Ochman et al. 2010; Degnan et al. 2012). This pattern of codiversification between hosts and their gut microbiota could stem from both heritable factors, such as host genetics and the vertical, generation-to-generation transmission of gut microbes (Vaishampayan et al. 2010), and environmental factors, such as host diet and geography (Ley et al. 2008a,b; Turnbaugh et al. 2008, 2009; De Filippo et al. 2010; La Serre et al. 2010; Muegge et al. 2011; Claesson et al. 2012; Yatsunenko et al. 2012). However, because the great ape species sampled to date represent populations that are at once phylogenetically, ecologically, and geographically distinct, it has not been possible to separate the relative influences of heritable and environmental factors on the evolution of the great ape gut microbiota.

One approach to parsing the effects of environmental factors on the gut microbiota from those of heritable factors is to compare sympatric (i.e., co-occurring) and allopatric (i.e., geographically separated) host populations. Gorillas diverged from the lineage leading to humans and chimpanzees/bonobos at least 6 million years ago (Glazko and Nei 2003; Langergraber et al. 2012), but since that time, the two groups have come into secondary contact

\footnotetext{
${ }^{5}$ Corresponding author

E-mail howard.ochman@yale.edu

Article published online before print. Article, supplemental material, and publication date are at http://www.genome.org/cgi/doi/10.1101/gr.154773.113.
}

throughout equatorial Africa. When living in sympatry, great ape species experience dietary convergence in addition to shared geography (Williamson et al. 1990; Tutin and Fernandez 1993; Shannon et al. 2006; Yamagiwa and Basabose 2006), but they do not mingle or interbreed, and their phylogenetic distinctiveness is maintained. Therefore, the effects on the gut microbiota of the environmental factors shared exclusively by sympatric chimpanzees and gorillas can be measured quantitatively as the degree of compositional convergence between the gut microbiota of sympatric populations relative to those of allopatric populations.

To quantify the effects of shared environmental factors on the gut microbiota of sympatric great apes, we have investigated the gut microbiota of sympatric and allopatric populations of chimpanzees, bonobos, and gorillas from Tanzania, Cameroon, the Central African Republic (CAR), and the Democratic Republic of the Congo (DRC). We show that, while hosts of different species always maintain distinct gut microbiota (even when living in sympatry), the gut microbiota of sympatric Pan and Gorilla share significantly more bacterial phylotypes than do those of allopatric Pan and Gorilla. Moreover, the specific patterns of phylotype sharing indicate a history of transfer of gut bacteria between the two host species, with chimpanzees acquiring bacteria from sympatric gorillas. Recent analyses of human populations have shown how geographic factors can shape intraspecific variation in gut microbiota composition (De Filippo et al. 2010; Yatsunenko et al. 2012); our results broaden this principle to include a role for geographic isolation in maintaining differences in gut microbiota composition among closely related heterospecific hosts. 


\section{Results}

\section{Sample sources}

We assayed the gut microbial communities, as present in fecal samples, of gorilla subspecies Gorilla gorilla gorilla (western lowland gorilla) from Cameroon and the CAR, and of gorilla subspecies Gorilla beringei graueri (eastern lowland gorilla) from the DRC. These data were merged with sequences generated previously from the gut microbiomes of chimpanzee subspecies Pan troglodytes ellioti from Cameroon, Pan troglodytes troglodytes from Cameroon, Pan troglodytes schweinfurthii from Tanzania, and Pan paniscus from the DRC (Ochman et al. 2010; Degnan et al. 2012), yielding a data set containing 1,321,276 processed reads and averaging 15,544 reads per sample. These reads clustered into 2,392 99\% operational taxonomic units (OTUs) (i.e., phylotypes). A map of field collection sites is presented in Figure 1, and detailed sample information is presented in Supplemental Table S1. Altogether, the sample set allowed comparisons of one pair of sympatric heterogeneric populations (G. g. gorilla with $P$. t. troglodytes), one pair of neighboring heterogeneric populations (G. g. gorilla with $P$. t. ellioti), and six pairs of geographically separated heterogeneric populations (G. g. gorilla with P. paniscus, G. b. graueri with P. paniscus, G. g. gorilla with P. t. schweinfurthii, G. b. graueri with P. t. ellioti, G. b. graueri with P. t. troglodytes, and G. b. graueri with P. t. schweinfurthii).

\section{Sympatry leads to convergence in gut microbial community constituents}

To evaluate the effects of shared environmental factors on the composition of gut microbial communities of sympatric chimpanzees and gorillas, we tested whether the microbiota of chimpanzees and gorillas living in sympatry shared more phylotypes with each other than did the microbiota of geographically isolated heterogeneric populations. Pairwise binary Bray-Curtis dissimilarities, which reflect the similarity between communities in terms of the presence/absence of bacterial phylotypes, and unweighted UniFrac distances, which reflect the similarity between communities in terms of the overlap between their phylogenetic trees, indicated that the microbiota of western lowland gorillas (G.g. gorilla) were significantly more similar to the microbiota of chimpanzees with which they live sympatrically (P. t. troglodytes) than they were to the microbiota of either allopatric chimpanzees from Tanzania (P. t. schweinfurthii) or allopatric bonobos from the DRC (P. paniscus) ( $t$-tests, $P<0.01$ ) (Figs. 2,3$)$. Moreover, the gut microbiota of sympatric chimpanzees and gorillas were more similar to one another than were the gut microbiota of either allopatric bonobos and eastern lowland gorillas or allopatric chimpanzees from Tanzania and eastern lowland gorillas ( $t$-tests, $P<0.01$; Figs. 2,3 ). On average, western lowland gorillas shared 53\% more bacterial phylotypes with sympatric chimpanzees than they did with isolated chimpanzees. In these comparisons, geographic isolation reduced the number of phylotypes shared between heterogeneric species by an average of $35 \%$. A Venn diagram displaying the phylotype sharing among hosts is presented in Supplemental Figure S1.

In contrast, the gut microbiota of sympatric chimpanzees and gorillas were no more similar to one another than the gut microbiota of neighboring chimpanzees and gorillas (P. t. ellioti and G. $g$. gorilla; $t$-test, $P>0.05$ ), and the gut microbiota of Cameroon chimpanzees were no more similar to the gut microbiota of sympatric western lowland gorillas than they were to the gut microbiota of eastern lowland gorillas, which live sympatrically with unsampled populations of chimpanzees in the DRC ( $t$-test, $P>0.05$ ) (Figs. 2,3 ).

\section{Maintenance of species-specific gut microbiota in sympatric populations}

Next, we evaluated the effects of host phylogenic history on the composition of the gut microbiota of chimpanzees and gorillas while controlling for host geography. Pairwise binary Bray-Curtis dissimilarities and unweighted UniFrac distances indicated that the microbiota of each pair of congeneric populations were more similar to one another than were the microbiota of each pair of heterogeneric populations ( $t$-tests, $P<0.01$ ) (Figs. 2,3$)$. In addition, a parsimony reconstruction of the relationships among great ape gut microbiota based on the log abundances of bacterial phylotypes revealed that the microbiota of hosts of different species could always be distinguished from one another (Supplemental Fig. S2). On average, isolated congenerics shared $84 \%$ more bacterial phylotypes than did sympatric heterogenerics and $182 \%$ more bacterial phylotypes than did allopatric heterogenerics.

\section{Convergence of the relative abundances of phylotypes within sympatric great ape gut microbiomes}

In addition to measuring the effect of shared environmental factors on the presence/absence of bacterial phylotypes within the gut microbiota of sympatric chimpanzees and gorillas, we evaluated the effect on the relative abundances of bacterial phylotypes using several metrics (Euclidean distance, Pearson distance, and weighted Unifrac distance). These metrics revealed qualitatively similar patterns of similarities and differences among great ape gut communities as did the measures
Figure 1. Map of ape species ranges and fecal collection sites. Circles and squares denote Pan and Gorilla collection sites, respectively. Composite shapes denote locations where fecal samples from both host genera were collected. Colored borders represent geographic ranges of their respective host populations.

\section{Genome Research \\ www.genome.org}




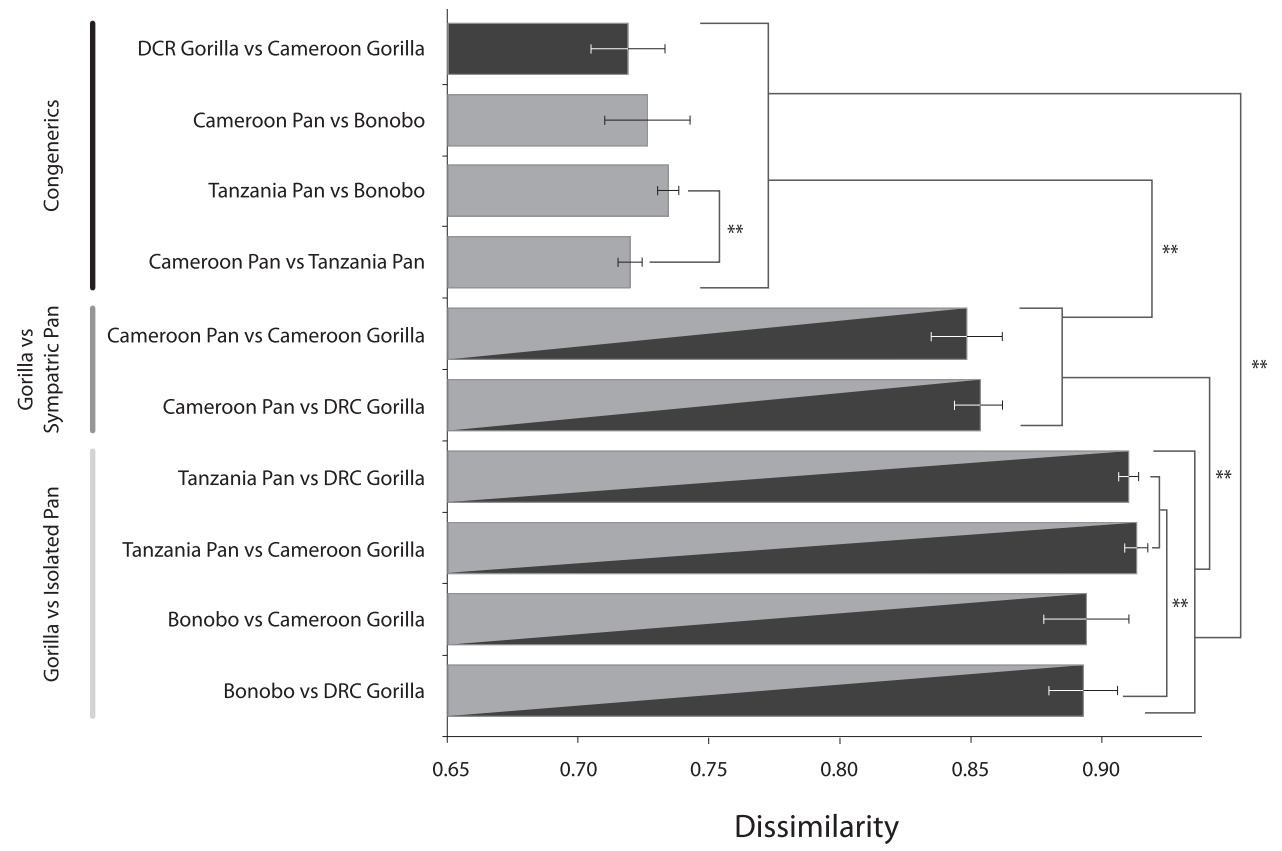

Figure 2. Convergence of gut microbiota of sympatric chimpanzees and gorillas. Horizontal bars show average pairwise binary Bray-Curtis dissimilarities between the gut microbiota of individuals from pairs of host populations. Bars are shaded to represent the hosts being compared (black, Gorilla; gray, Pan), with striped bars representing comparisons between Gorilla and Pan, labeled to reflect whether they live sympatrically or allopatrically. Asterisks denote level of statistical significance; Bonferroni-corrected $P<0.01$.

based on the presence/absence of bacterial phylotypes, supporting both the convergence of the gut microbiota of sympatric chimpanzees and gorillas and the maintenance of distinct gut microbiota in each host species (Supplemental Fig. S3).

Taxonomic profiles of bacterial phylotypes shared exclusively by sympatric chimpanzees and gorillas

To determine the phylogenetic distribution of bacterial phylotypes whose occurrence patterns were associated with the shared environment of sympatric hosts, we evaluated the taxonomic classifications of the phylotypes recovered only from sympatric chimpanzees and gorillas. These phylotypes spanned the most common phyla of great ape gut bacteria (Bacteroidetes, Firmicutes, Proteobacteria, and Actinobacteria) and principally represented seven taxonomic orders. The average relative abundances of these phylotypes at the order level within sympatric chimpanzees and gorillas are presented in Supplemental Figure S4. The specific phylotypes shared exclusively by sympatric chimpanzees and gorillas, and their relative abundances across hosts, are presented in Supplemental Table S2.
A

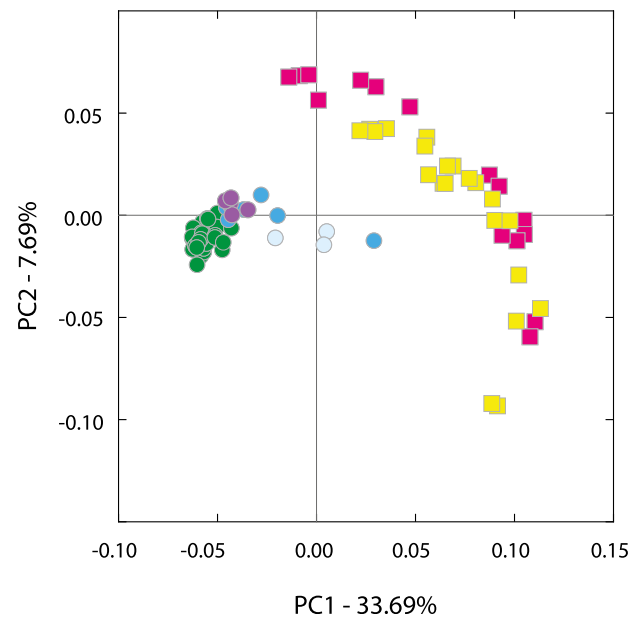

B

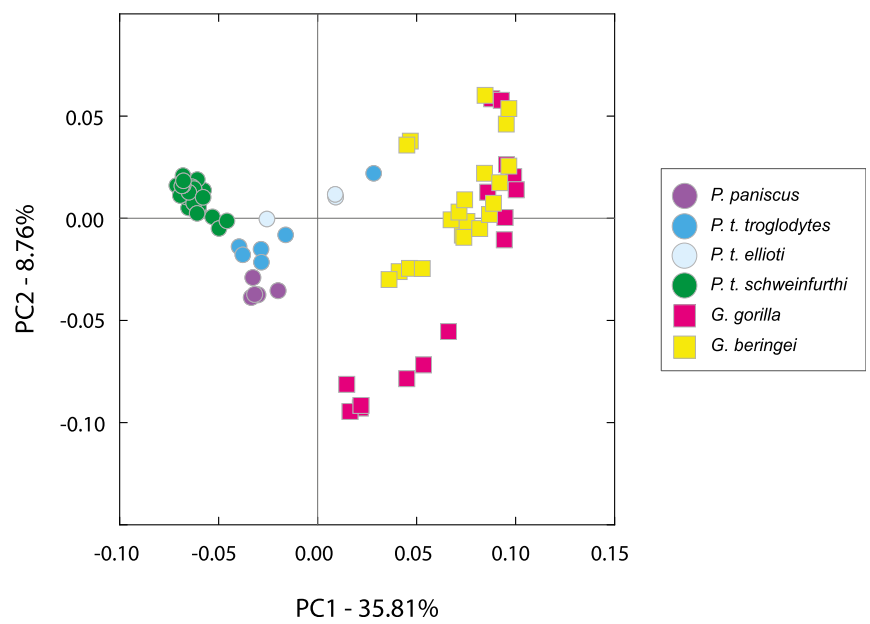

Figure 3. Increased sharing of bacterial phylotypes by sympatric hosts. Principal coordinates plots show the first two principal coordinates of the pairwise binary Bray-Curtis dissimilarities $(A)$ and unweighted UniFrac distances $(B)$ among hosts. Shown are the mean values for each sample calculated from rarefaction analyses. Samples are colored by the population from which they were collected corresponding to Figure 1. Note that sympatric Pan individuals cluster more closely with Gorilla individuals than do isolated Pan individuals. 


\section{Discussion}

Comparisons of sympatric and allopatric populations of the genera Pan and Gorilla allowed us to quantify the relative influences of heritable and environmental factors on the composition of great ape gut microbial communities. First, it is noteworthy that chimpanzees, bonobos, and gorillas could always be distinguished from one another by the composition of their gut microbiota (Supplemental Fig. S2) and that congeneric hosts shared, on average, more than twice as many bacterial phylotypes as hosts from different genera (Figs. 2, 3). Despite the strong influence of host phylogenetic history on the gut microbiota, the gut microbiota of Pan and Gorilla converged where the two genera are sympatric: The environmental factors experienced exclusively by sympatric chimpanzees and gorillas increased the number of phylotypes shared between these host genera by an average of $\sim 53 \%$ (Figs. 2,3 ) and led to the convergence of the relative abundances of bacterial taxa within their gut microbiota (Supplemental Fig. S3).

The phylotypes shared exclusively by hosts living in sympatry spanned the four major phyla of gut bacteria (i.e., Bacteroidetes, Firmicutes, Proteobacteria, and Actinobacteria) and some of the most common taxonomic orders (e.g., Bacteroidales and Clostridiales) (Supplemental Fig. S4). Given that many of these phylotypes are not typically found outside mammalian GI tracts (Ley et al. 2008b), one explanation for the convergence of the gut microbiota of sympatric chimpanzees and gorillas is that phylotypes have been transferred between hosts of different species. That the microbiota of chimpanzees in Cameroon were equally similar to those of western and eastern lowland gorillas is consistent with the hypothesis that Cameroon chimpanzees acquired gut bacteria derived from gorillas. An alternative, but less parsimonious, explanation is that the environmental conditions that facilitate chimpanzees living in sympatry with gorillas also promoted the occurrence of bacterial phylotypes not favored by environments in which chimpanzees live in isolation from gorillas.

Proximity between hosts has been linked to the exchange of gut microbes within species: In humans, the microbiota of unrelated hosts living in the same household tend to have more similar sets of bacterial phylotypes than do those of unrelated hosts of different households (Yatsunenko et al. 2012), and in experiments with mice, hosts reared in the same cage tend to share gut bacteria (Campbell et al. 2012). Our results show that the homogenizing effect of cohabitation extends to the gut microbiota of different host species in their natural environments.

It is possible for great ape species occupying the same range to experience incidental contact, thereby providing a route for microbes to be transferred directly among individuals. Sympatric chimpanzees and gorillas have been observed feeding concurrently at the same tree crown, sometimes as closely as a few meters from one another (Shannon et al. 2006), and proximity between hosts has been implicated in the transfer of simian immunodeficiency virus (SIV) as well as hepatitis B virus from wild chimpanzees to gorillas (Takehisa et al. 2009). However, because direct contact between chimpanzees and gorillas is rare, even when the hosts co-occur, it is more likely that microbes are transferred between host species via indirect mechanisms, such as the acquisition of microbes from fecal contamination in the environment. The high degree of phylotype sharing by neighboring populations of G. g. gorilla and P. t. ellioti, whose ranges are adjacent but separated by the Sanaga River, indicates that bacterial transfers may occur even if hosts do not come into direct contact.
Recent surveys of microbial communities indicate there are barriers to bacterial dispersal that can restrict strains to particular locations (Foissner 2006; Green and Bohannan 2006; Martiny et al. 2006; Ramette and Tiedje 2007; Lindstrom and Langenheder 2011; Hanson et al. 2012), potentially hindering the transfer of microbes between host species that are geographically isolated. That the influence of shared environmental factors on the microbiota of sympatric chimpanzees and gorillas was evident in the occurrence patterns of $99 \%$ similarity bacterial phylotypes within bacterial taxa shared by all populations of great apes lends support to this view (Supplemental Fig. S4). For example, sympatric chimpanzees and gorillas in Cameroon possessed identical phylotypes from the genus Prevotella, whereas the chimpanzees from Tanzania housed a nonoverlapping set of Prevotella lineages. These results suggest that phylotypes within many of the dominant taxa of gut bacteria are confined to specific geographic locations.

Dietary overlap between hosts is another possible source of the convergence of the microbiota of chimpanzees and gorillas living in sympatry. Host diet influences the gut microbiota in terms of both the presence/absence and the relative abundances of bacterial phylotypes (Ley et al. 2008a,b; Turnbaugh et al. 2008, 2009; De Filippo et al. 2010; La Serre et al. 2010; Muegge et al. 2011; Claesson et al. 2012; Ravussin et al. 2012; Serion et al. 2012). Additionally, the gut enterotypes that have been identified in humans and chimpanzees based on the relative abundances of bacterial genera (Arumugam et al. 2011; Wu et al. 2011; Moeller et al. 2012; Qin et al. 2012) appear to be influenced by the long-term diet of the host (Wu et al. 2011). The diets of sympatric chimpanzees and gorillas can include up to $80 \%$ of the same food species, many of which are not shared when the host species live allopatrically (Williamson et al. 1990; Tutin and Fernandez 1993). Chimpanzees and gorillas living in sympatry may therefore cultivate similar gut environments due to their shared diets, potentially favoring specific bacterial constituents.

Consistent with previous results suggesting a history of codiversification between great apes and their microbiota (Ochman et al. 2010; Degnan et al. 2012), the microbiota of congeneric populations were always more similar in terms of the presence/ absence and relative abundances of bacterial phylotypes than were those of heterogeneric populations (Figs. 2, 3; Supplemental Fig. S3), and the microbiota of different host species were always distinguishable from one another (Supplemental Fig. S2). The uneven distribution of phylotypes among chimpanzees, bonobos, and gorillas is consistent with a pattern of vertical transmission over evolutionary timescales and suggests that many of the phylotypes within great ape gut microbiomes are specifically adapted to their respective host species.

Our data indicate important roles for both heritable and environmental factors in determining the composition of great ape gut microbiota. However, not all variation in gut microbiota composition could be explained by host phylogeny or geography, as several phylotypes were detected only in allopatric, heterogeneric host populations. For example, Tanzania chimpanzees and allopatric western lowland gorillas shared 12 bacterial phylotypes that were never recovered from any other host populations (Supplemental Fig. S1). These results reflect the intricacy of the many interacting factors that determine gut microbiota composition.

In sum, comparing sympatric and allopatric host populations enabled us to tease apart the influences of heritable and environmental factors on the evolution of great ape gut microbial communities. These results illustrate how sampling schemes designed to parse potentially correlated host features, such as geographic and phylogenetic divergence, can provide nuanced insights into

\section{Genome Research}

www.genome.org 
the manner by which gut microbes assort among host species. The convergence observed in sympatric populations relative to allopatric populations implies that evolutionary differentiation of great ape gut microbiomes has been maintained by the geographic isolation among host species.

\section{Methods}

\section{Sample preparation}

Gorilla fecal samples were collected from field sites in Cameroon, the CAR, and the DRC, preserved in RNAlater (Ambion), and stored at $-80^{\circ} \mathrm{C}$. For each sample, host species was determined by sequencing of the D-loop of the host mitochondrial genome. To assay the microbial diversity within each fecal sample, the total DNA from $50 \mu \mathrm{L}$ aliquots of thawed sample was extracted by a bead-beating procedure according to the method previously described (Goodman et al. 2011). PCR amplifications of the $16 \mathrm{~S}$ rDNA region encompassed by primers $926 \mathrm{~F}$ and $1492 \mathrm{R}$ were performed according to the method previously described (Ochman et al. 2010), and the resulting amplicons were subjected to pyrosequencing on a 454 Life Sciences (Roche) GS FLX Titanium instrument.

\section{Sequence filtering}

Pyrosequencing reads were processed in QIIME version 1.5.0 (Caporaso et al. 2010). Flowgrams were obtained in SFF file format and converted to FASTA and QUAL files with process_sff.py. Reads were assigned to their respective samples based on their identifying barcode with split_libraries.py, allowing a minimum quality score of 28 and no errors in the barcode. Reads attaining these quality standards were assigned to 99\% OTUs with pick_otus.py using the $u$ clust algorithm and classified with assign_taxonomy.py using the RDP classifier. An OTU table was produced with make_otu_table.py. To eliminate pyrosequencing errors and to focus our analyses on primary constituents of the gut communities, the OTU table was filtered to remove all OTUs represented by fewer than 50 reads. The OTU table, including taxonomic classifications for each OTU, is presented in Supplemental Table S3. A FASTA file containing a representative sequence for each OTU is presented in the Supplemental Methods.

\section{Microbial community relationships within and among host species}

To evaluate the effects of environmental and heritable factors on the gut microbiota, pairwise binary Bray-Curtis dissimilarities, Euclidean distances, Pearson distances, and both weighted and unweighted UniFrac distances between all pairs of samples were calculated with beta_diversity.py in QIIME version 1.5.0 from 100 rarefied OTU tables each subsampled to 5545 reads. Rarefaction analyses are presented in Supplemental Figure S5. Two-tailed $t$-tests were performed to test for differences between comparisons of the gut microbiota of pairs of host populations (e.g., "Cameroon chimpanzee vs. western lowland gorilla" vs. "Tanzania chimpanzee vs. western lowland gorilla"). Figure 2 displays results calculated from a single rarefied OTU table, but all replicate rarefied OTU tables produced qualitatively similar results. We observed a strict significance threshold of Bonferroni-corrected $P$-values $<0.01$. Principal coordinates plots were generated in QIIME version 1.5.0.

\section{Taxonomic profiles of phylotypes shared by sympatric chimpanzees and gorillas}

Taxonomic classifications of all phylotypes shared by sympatric chimpanzees and gorillas were used to generate order-level abundance distributions of shared phylotypes for each host species. These abundance distributions were then used to create pie charts in QIIME version 1.5.0.

\section{Data access}

Sequence data generated for this study have been submitted to the NCBI Sequence Read Archive (SRA; http://www.ncbi.nlm.nih.gov/ sra) under accession number SRR799915.

\section{Acknowledgments}

We thank Kim Hammond for assistance with the preparation of figures; Eitel Mpoudi Ngolle and the SIV team of Project PRESICA for field work and logistical support in Cameroon; the Cameroonian Ministries of Health, Forestry and Wildlife, and Research for permission to collect samples in Cameroon; the Water and Forest Ministry for permission to collect samples in the Central African Republic; the Ministry of Scientific Research and Technology, and the Department of Ecology and Management of Plant and Animal Resources of the University of Kisangani for permission to collect samples in the DRC. This work was supported by grants from the National Institutes of Health (R01 AI50529, R01 AI58715 to B.H.H. and R01 GM101209 to H.O.), the Agence Nationale de Recherche sur le Sida (12152/12182), and a National Science Foundation Graduate Research Fellowship to A.H.M.

\section{References}

Arumugam M, Raes J, Pelletier E, Le Paslier D, Yamada T, Mende DR, Fernandes GR, Tap J, Bruls T, Batto JM, et al. 2011. Enterotypes of the human gut microbiome. Nature 473: 174-180.

Campbell JH, Foster CM, Vishnivetskaya T, Campbell AG, Yang ZK, Wymore A Palumbo AV, Chesler EJ, Podar M. 2012. Host genetic and environmental effects on mouse intestinal microbiota. ISME J 11: 2033-2044.

Caporaso JG, Kuczynski J, Strombaugh J, Bittinger K, Bushman FD, Costello EK, Fierer N, Pena AG, Goodrich JK, Gordon JI. 2010. QIIME allows analysis of high-throughput community sequencing data. Nat Methods 7: $335-336$.

Claesson MJ, Jeffrey IB, Conde S, Power SE, O'Conner EM, Cusack S, Harris HM, Coakley M, Lakshminarayanan B, O'Sullivan O, et al. 2012. Gut microbiota composition correlates with diet and health in the elderly. Nature 488: 178-184.

De Filippo C, Cavalieri D, Paola MD, Ramazzotti M, Poullet JB, Massart S, Collini S, Pieraccini G, Lionetti P. 2010. Impact of diet in shaping gut microbiota revealed by a comparative study in children from Europe and rural Africa. Proc Natl Acad Sci 107: 14691-14696.

Degnan PH, Pusey AE, Wilson ML, Lonsdorf EV, Goodall J, Rudicell RS, Wroblewski EE, Hahn BH, Ochman H. 2012. Factors responsible for the diversification of the gut microbial communities within chimpanzees from Gombe National Park. Proc Natl Acad Sci 109: 13034-13039.

Foissner W. 2006. Biogeography and dispersal of micro-organisms: A review emphasizing protists. Acta Protozool 2: 111-136.

Glazko GV, Nei M. 2003. Estimation of divergence times for major lineages of primate species. Mol Biol Evol 3: 424-434.

Goodman AL, Kallstrom G, Faith JJ, Reyes A, Moore A, Dantas G, Gordon JI. 2011. Extensive personal human gut microbiota culture collections characterized and manipulated in gnotobiotic mice. Proc Natl Acad Sci 108: 6252-6257.

Green J, Bohannan BJM. 2006. Spatial scaling of microbial biodiversity Trends Ecol Evol 9: 501-507.

Hanson CA, Fuhrman JA, Horner-Devine MC, Martiny JBH. 2012. Beyond biogeographic patterns: Processes shaping the microbial landscape. Nat Rev Microbiol 10: 497-506.

La Serre CB, Ellis CL, Lee J, Hartman AL, Rutledge JC, Raybould HE. 2010. Propensity to high-fat diet-induced obesity in rats is associated with changes in the gut microbiota and gut inflammation. Horm Signal 2: 440-448.

Langergraber KE, Prufer K, Rowney C, Boesch C, Crockford C, Fawcett K, Inoue E, Inoue-Muruyama M, Mitani JC, Muller MN. 2012. Generation times in wild chimpanzees and gorillas suggest earlier divergence times in great ape and human evolution. Proc Natl Acad Sci 109: 15716-15721. 
Ley RE, Hamady M, Lozupone C, Turnbaugh PJ, Ramey RR, Bircher SJ, Schlegel ML, Tucker TA, Schrenzel MD, Knight R, et al. 2008a. Evolution of mammals and their gut microbes. Science 320: 1647-1651.

Ley RE, Lozupone CA, Hamady M, Knight R, Gordon JI. 2008b. Worlds within worlds: Evolution of the vertebrate gut microbiota. Nat Rev Microbiol 6: 776-788.

Lindstrom ES, Langenheder S. 2011. Local and regional factors influencing bacterial community assembly. Environ. Microbiol. Reports 1: 1-9.

Martiny JBH, Bohannan BJM, Brown JH, Colwell RK, Fuhrman JA, Green JL, Horner-Devine MC, Kane M, Krumins JA, Kuske CR. 2006. Microbial biogeography: Putting microorganisms on the map. Nat Rev Microbiol 4: $102-112$.

Moeller AH, Degnan PH, Pusey A, Wilson M, Hahn BH, Ochman H. 2012. Chimpanzees and humans harbour compositionally similar gut enterotypes. Nature Comm 3: 1179-1184.

Muegge BD, Kuczynski J, Knights D, Clemente JC, González A, Fontana L, Henrissat B, Knight R, Gordon JI. 2011. Diet drives convergence in gut microbiome functions across mammalian phylogeny and within humans. Science 332: 970-974.

Ochman H, Worobey M, Kuo CH, Ndjango JN, Peeters M, Hahn BH, Hugenholtz P. 2010. Evolutionary relationships of wild hominids recapitulated by gut microbial communities. PLoS Biol 11: e1000546.

Qin J, Li Y, Cai Z, Li S, Zhu J, Zhang F, Liang S, Zhang W, Guan Y, Shen D. 2012. A metagenome-wide association study of gut microbiota in type 2 diabetes. Nature 490: 55-60.

Ramette A, Tiedje JM. 2007. Multiscale responses of microbial life to spatial distance and environmental heterogeneity in a patchy ecosystem. Proc Natl Acad Sci 104: 2761-2766.

Ravussin Y, Koren O, Spor A, LeDuc C, Gutman R, Stombaugh J, Knight R, Ley RE, Leibel RL. 2012. Responses of gut microbiota to diet composition and weight loss in lean and obese mice. Integrative Physiol. 4: 738-747.

Serion M, Luche E, Gres S, Baylac A, Berge M, Cenac C, Waget A, Klopp P, Iacovoni J, Klopp C, et al. 2012. Metabolic adaptation to a high-fat diet is associated with a change in the gut microbiota. Gut 61: $543-553$.
Shannon P, Markiel A, Ozier O, Baliga NS, Wang JT, Ramage D, Amin N, Stanford CB. 2006. The behavioral ecology of sympatric African apes: Implications for understanding fossil hominoid ecology. Primates 1: 91-101.

Takehisa J, Kraus MH, Ayouba A, Bailes E, Heuverswyn FV, Decker JM, Li Y, Rudicell RS, Learn GH, Neel C, et al. 2009. Origin and biology of simian immunodecifiency virus in wild-living western gorillas. J Virol 4: 1635.

Turnbaugh PJ, Backhead F, Fulton L, Gordon JI. 2008. Diet-induced obesity is linked to marked but reversible alterations in the mouse distal gut microbiome. Cell Host Microbe 4: 213-223.

Turnbaugh PJ, Riduara VK, Faith JJ, Rey FE, Knight R, Gordon JI. 2009. The effect of diet on the human gut microbiome: A metagenomic analysis in humanized gnotobiotic mice. Sci Transl Med 6: 6-14.

Tutin CEG, Fernandez M. 1993. Composition of the diet of chimpanzees and comparisons with that of sympatric lowland gorillas in the lope reserve, Gabon. Am J Primatol 3: 195-211.

Vaishampayan PA, Kuehl JV, Froula JL, Morgan JL, Ochman H, Francino MP. 2010. Comparative metagenomics and population dynamics of the gut microbiota in mother and infant. Genome Biol Evol 2: 53-66.

Williamson EA, Tutin CE, Rogers ME, Fernandez M. 1990. Composition of the diet of lowland gorillas at Lope in Gabon. Am J Primatol 4: $265-277$.

Wu GD, Chen J, Hoffmann C, Bittinger K, Chen YY, Keilbaugh SA, Bewtra M, Knights D, Walters WA, Knight R, et al. 2011. Linking long-term dietary patterns with gut microbial enterotypes. Science 334: 105-108.

Yamagiwa J, Basabose AK. 2006. Diet and seasonal changes in sympatric gorillas and chimpanzees at Kahuzi-Biega National Park. Primates 1: 74-90.

Yatsunenko T, Rey FE, Manary MJ, Trehan I, Dominguez-Bello MG, Contreras M, Magris M, Hidalgo G, Baldassano RN, Anokhin AP, et al. 2012. Human gut microbiome viewed across age and geography. Nature 486: $222-227$.

Received January 10, 2013; accepted in revised form June 19, 2013.

\section{Genome Research




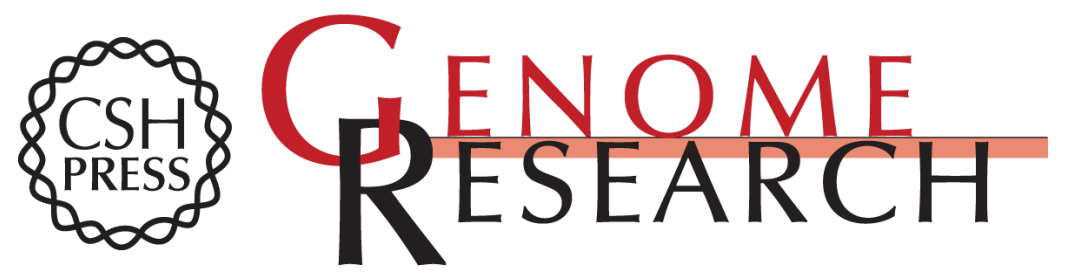

\section{Sympatric chimpanzees and gorillas harbor convergent gut microbial communities}

Andrew H. Moeller, Martine Peeters, Jean-Basco Ndjango, et al.

Genome Res. 2013 23: 1715-1720 originally published online June 26, 2013

Access the most recent version at doi:10.1101/gr.154773.113

Supplemental Material

References

Creative

Commons

License

Email Alerting

Service
http://genome.cshlp.org/content/suppl/2013/08/06/gr.154773.113.DC1

This article cites 34 articles, 9 of which can be accessed free at: http://genome.cshlp.org/content/23/10/1715.full.html\#ref-list-1

This article is distributed exclusively by Cold Spring Harbor Laboratory Press for the first six months after the full-issue publication date (see

http://genome.cshlp.org/site/misc/terms.xhtml). After six months, it is available under a Creative Commons License (Attribution-NonCommercial 3.0 Unported), as described at http://creativecommons.org/licenses/by-nc/3.0/.

Receive free email alerts when new articles cite this article - sign up in the box at the top right corner of the article or click here.

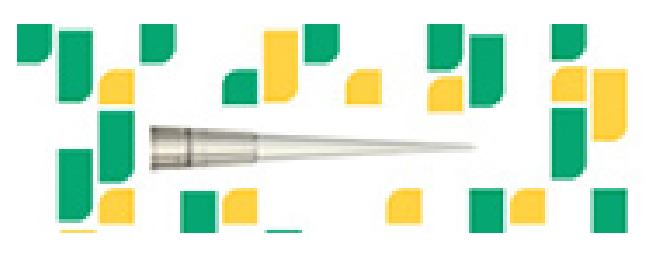

Focused on your science.

Jコగ

SCIENTIFIC

saos or seisnes

To subscribe to Genome Research go to: https://genome.cshlp.org/subscriptions 